\title{
Copper indium sulphide:zinc sulphide (CIS:ZnS)-alloyed quantum dots as an eco-friendly absorber in solar cells
}

\author{
M SENTHILKUMAR, C IMLA MARY, M PANDIYARAJAN, G MANOBALAJI \\ and S MOORTHY BABU* \\ Crystal Growth Centre, Anna University, Chennai 600025, India \\ *Author for correspondence (babusm@yahoo.com; babu@annauniv.edu)
}

MS received 15 October 2018; accepted 26 March 2019

\begin{abstract}
Synthesis and characterization of less toxic copper indium zinc sulphide (CIS:ZnS)-alloyed quantum dots (QDs) were carried out and the ligand exchange process towards the efficiency enhancement in CIS:ZnS QD-sensitized solar cell was demonstrated. The colloidal CIS:ZnS QDs were synthesized by an inexpensive heat up method with oleic acid as the capping ligand. The optical properties were analysed through ultraviolet-visible absorption and photoluminescence emission spectroscopy. The influence of the ligand exchange process on the CIS:ZnS QD-based solar cells was analysed with the fabrication of two batches of solar cells. The ligand exchange process was confirmed from Fourier transform infrared and thermogravimetric analyses. The QD-sensitized solar cells were fabricated using a CIS:ZnS QD-loaded titania photoanode and by employing copper sulphide as the counter electrode. The photovoltaic performance of the fabricated QD solar cells was analysed through photovoltaic characterization methods (current density-voltage characteristics of the devices under the simulated solar light conditions and external quantum efficiency measurements). The ligand-exchanged QD-loaded solar cells show enhanced power conversion efficiency compared to the long chain ligand-capped CIS:ZnS QD-sensitized solar cells.
\end{abstract}

Keywords. Copper indium zinc sulphide; less hazardous QDs; oleic acid; ligand exchange; QD-sensitized solar cells; efficiency enhancement.

\section{Introduction}

Semiconducting quantum dots (QDs) exhibit interesting photo-physical and chemical properties, especially, the sizeand shape-dependent band gap tunability of QDs facilitates their application in the field of photocatalysis [1], photovoltaics [2], sensors [3], light emitting devices [4], thermoelectrics [5], bioimaging [6], photothermal therapy [7], solar concentrators [8], etc. However, the conventional heavy metal-based (cadmium and lead) QDs are highly toxic and the environmental restriction limits the commercialization of these kinds of material-based devices [9]. Hence, discovering a hazardless QD material analogues to the conventional heavy metal (cadmium, lead and mercury)-based QDs has been a compelling research focus in the last few decades. However, recently emerging multinary metal chalcogenide materials outperform some of the properties of conventional QDs, but there are many research problems yet to be addressed. In the field of next generation solar cells, copper- and silverbased multinary QDs attracted significant attention in the last few decades as an inexpensive, harmless alternative to heavy metal-based semiconducting materials $[10,11]$. A copper indium sulphide:zinc sulphide-alloyed (abbreviated as CIS:ZnS or simply CIZS) QD is one of the interesting materials owing to its size- and shape-dependent tunability of its optical, structural and photo-physical properties. The optical absorption of these CIS:ZnS QDs can be tuned from the visible to near infrared region which implies the quality of these materials to be utilized for solar cell applications.

Solution-processed colloidal methods are the most versatile technique which have been utilized for the synthesis of CIS:ZnS QDs with homogeneous size distribution $[12,13]$. However, long chain capping ligands were used for the synthesis of high-quality colloidal QDs. Unfortunately, these capping ligands turned as an insulating layer between the particles which hinders the charge carrier mobility and restricts the application of these QDs towards optoelectronic devices [14]. Hence, it is necessary to exchange the long chain capping ligands with short chain ligands, for the effective stabilization of QDs as well as, to reduce the insulation behaviour of the surface-passivated ligands. In this report, we have successfully synthesized environmentally benign CIS:ZnS multinary QDs with high photoluminescence (PL) properties and the fabrication of CIS:ZnS QD-sensitized solar cells was demonstrated. The long chain oleic acid (OLA) capping ligand, used during the synthesis of CIS:ZnS QDs for the reduction of the particle size and for stabilizing the QDs in solution, acts as an insulator for the charge carrier transportation. Hence, the native ligand (OLA) was replaced with a short chain capping ligand, 3-mercaptopropionic acid (MPA), without altering 
the features of the CIS:ZnS QDs. The QD-sensitized solar cells were fabricated by employing both kinds of CIS:ZnS (with and without ligand exchanged) QD-sensitized photoanodes and the influence of ligand exchange on the solar cell performance was investigated. The device performance was analysed by current density-voltage $(J-V)$ characteristics and by the incident photon to current conversion efficiency (IPCE) measurements.

\section{Experimental}

\subsection{Synthesis of colloidal CIS:ZnS QDS}

The CIS:ZnS QDs were synthesized by a simple heat up method using OLA as the capping ligand and 1-octanethiol (OCT) was employed as the sulphur source. Copper iodide $(\mathrm{CuI})$, zinc acetate $\left(\mathrm{Zn}\left(\mathrm{O}_{2} \mathrm{CCH}_{3}\right)_{2}\right)$ and indium acetate $\left(\mathrm{In}\left(\mathrm{C}_{2} \mathrm{H}_{3} \mathrm{O}_{2}\right)_{3}\right)$ were taken as precursor materials. The precursors and the solvent ( $20 \mathrm{ml}$ of 1-octadecene) were added into a three necked flask which was connected with a Schlenk line setup and the reaction flask was provided with a nitrogen $\left(\mathrm{N}_{2}\right)$ atmosphere. Initially, the reaction flask was degassed for 15 min under vacuum at a temperature of $120^{\circ} \mathrm{C}$. Then, $4 \mathrm{ml}$ of OLA was injected into the system under ambient $\mathrm{N}_{2}$ followed by degassing for $15 \mathrm{~min}$. Then, $6 \mathrm{ml}$ of 1-OCT was injected into the flask and subsequently the degassing and the $\mathrm{N}_{2}$ purging steps were followed. Then, the reaction flask was heated to the appropriate heating condition $\left(\sim 230^{\circ} \mathrm{C}\right.$ under a $\mathrm{N}_{2}$ atmosphere) to form highly emissive CIS:ZnS QDs. Furthermore, the synthesized-colloidal QDs were purified several times by using toluene and ethanol to remove the unbound ligands and byproducts. Finally, the purified-CIS:ZnS QDs were stabilized in toluene for further characterization and device fabrication.

\subsection{Preparation of $Q D$-sensitized $\mathrm{TiO}_{2}$ photoanode}

Conducting glass substrates (fluorine-doped tin oxide-FTO) were used to coat the titanium dioxide $\left(\mathrm{TiO}_{2}\right)$ photoanode. Initially, the FTO substrates were cleaned thoroughly using soap solution, acetone, DI water and finally with ethanol. The blocking layer of $\mathrm{TiO}_{2}$ was coated on FTO substrates through the treatment with aqueous solution of $40 \mathrm{mM} \mathrm{TiCl}_{4}$ for $30 \mathrm{~min}$ at $80^{\circ} \mathrm{C}$. Then, the mesoporous $\mathrm{TiO}_{2}\left(\mathrm{mp}-\mathrm{TiO}_{2}\right)$ active layer was coated by a conventional doctor blade coating method using transparent titania paste (Sigma Aldrich) followed by annealing at $500^{\circ} \mathrm{C}$ for $1 \mathrm{~h}$. The area of the $\mathrm{TiO}_{2}$ active layer was fixed as $\sim 0.2 \mathrm{~cm}^{2}$ by masking the remaining area of the FTO glass plates with scotch tape. Furthermore, the scattering layer of $\mathrm{TiO}_{2}$ was coated by adapting the same method used for coating the mp- $\mathrm{TiO}_{2}$ layer. To ensure the uniform coverage of the blocking layer, the $\mathrm{TiCl}_{4}$ treatment was carried out again at $80^{\circ} \mathrm{C}$ for $30 \mathrm{~min}$.

\section{$2.3 Q D$ sensitization of $\mathrm{TiO}_{2}$ photoanode and ligand exchange processing}

The CIS:ZnS QDs were sensitized on a $\mathrm{TiO}_{2}$ photoanode by immersing the prepared $\mathrm{TiO}_{2}$ anodes into the QD solution for $48 \mathrm{~h}$ to ensure the maximum loading of QDs. The capping ligands which are stabilizing the QDs could be an insulating layer for the charge carriers to transport between the QDs. Hence, the original capping ligand (OLA) was replaced with MPA a shorter capping ligand. The ligand exchange process was carried out by immersing the QD (capped with a long chain ligand)-sensitized $\mathrm{TiO}_{2}$ photoanodes into the solution of a short chain ligand (MPA and methanol 2:10 v/v ratio) for 5 min followed by rinsing in methanol. Then the photoanodes were dried using $\mathrm{N}_{2}$ flow. For the comparison of the influence of ligand exchange on the solar cell performance, one batch of QD-loaded photoanodes was left without any ligand exchange process.

\subsection{Fabrication of CIS:ZnS QD-sensitized solar cells}

The CIS:ZnS QD-sensitized solar cells were fabricated by sandwiching the QD-sensitized $\mathrm{TiO}_{2}$ photoanode and the copper sulphide $\left(\mathrm{Cu}_{x} \mathrm{~S}\right)$ counter electrodes (CEs) together by the insertion of a drop of polysulphide electrolyte between these two electrodes. The $\mathrm{Cu}_{x} \mathrm{~S}$ CEs were prepared by the sulphurization of sputtered $\mathrm{Cu}$ thin films (the area of the $\mathrm{CE}$ is $\sim 0.25 \mathrm{~cm}^{2}$ ) coated on the FTO glass plates. The polysulphide electrolyte was prepared by $\left(\mathrm{S}^{-} / \mathrm{S}_{n}^{2-}\right)$ dissolving the equal molar ratio of elemental $\mathrm{S}$ and sodium sulphide $\left(\mathrm{Na}_{2} \mathrm{~S} \cdot 9 \mathrm{H}_{2} \mathrm{O}\right)$ in DI water through an ultrasonication process.

\subsection{Characterization of QDs and solar cell performance}

The colloidal QDs were characterized through ultravioletvisible (UV-Vis) light absorption spectroscopy and PL emission spectroscopy for analysing the optical properties of the CIS:ZnS-alloyed QDs. The photovoltaic performance of the CIS:ZnS QD-sensitized solar cells was analysed through a two-electrode system with a solar light simulator under 1 sun illumination conditions. The power density of the solar light simulator was $100 \mathrm{~W} \mathrm{~cm}^{-2}$ using a xenon lamp with the $\mathrm{AM}$ 1.5 globe filter. The photovoltaic performance ( $J-V$ characteristics) of the prepared solar cells was studied by the voltage sweeping from $0 \mathrm{~V}$ to the maximum open circuit potential value. The scan rate was fixed at $10 \mathrm{mV} \mathrm{s}^{-1}$. The IPCE of the devices was analysed by using a Newport Oriel/IPCE instrument by tuning the incident light wavelength ranging from 300 to $700 \mathrm{~nm}$. Fourier transform infrared (FTIR) spectroscopy and thermogravimetric (TG) analyses are performed to understand the influence of the ligand exchange process on the QD surface. 


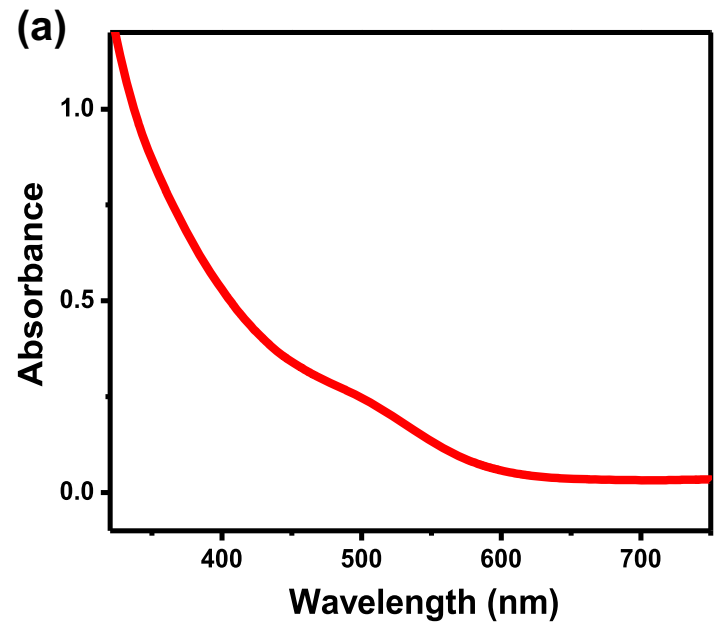

Figure 1. Optical properties of colloidal CIS:ZnS QDs. (a) UV-Vis absorption spectrum of the CIS:ZnS QDs and (b) band gap energy calculation.
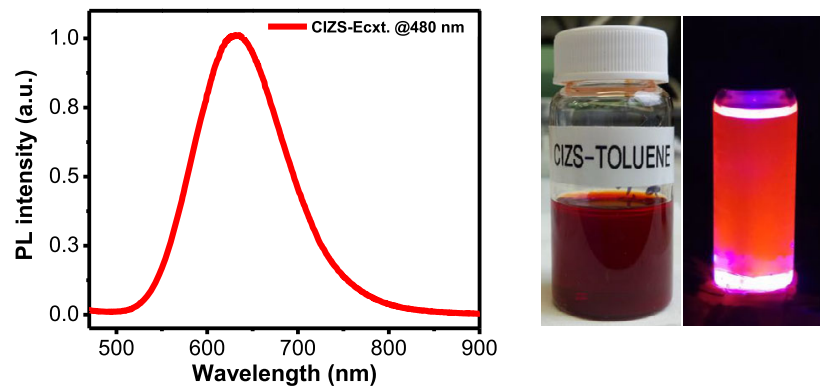

Figure 2. PL emission of the prepared CIS:ZnS QDs.

\section{Results and discussion}

The optical properties of the as-synthesized CIS:ZnS QDs were analysed through UV-Vis absorption and PL emission spectroscopy as shown in figures 1a, b and 2. CIS:ZnS QDs have broad absorption in the visible region and the absorption onset begins nearly at $600 \mathrm{~nm}$. The broad absorption feature is one of the characteristics of multinary QDs, which results from the discrete energy level distributions [2]. The band gap energy value $\left(E_{\mathrm{g}}\right)$ of the as-prepared colloidal CIS:ZnS QDs was calculated from the absorption spectrum through a Tauc's plot. Tauc's relationship for finding the band gap is

$$
E_{\mathrm{g}} \approx(\alpha h v)^{(1 / n)} / h v
$$

Here, $E_{\mathrm{g}}$ is the band gap energy, $h v$ is the energy of the light photon, $\alpha$ is the absorption coefficient of QDs, $h$ is Planck's constant and $n$ determines the nature of the electronic transition.

The band gap energy of the as-synthesized QDs was calculated by plotting $(\alpha h v)^{2}$ to the photon energy $(h v)$ and the linear extrapolation to the photon energy implies the band gap
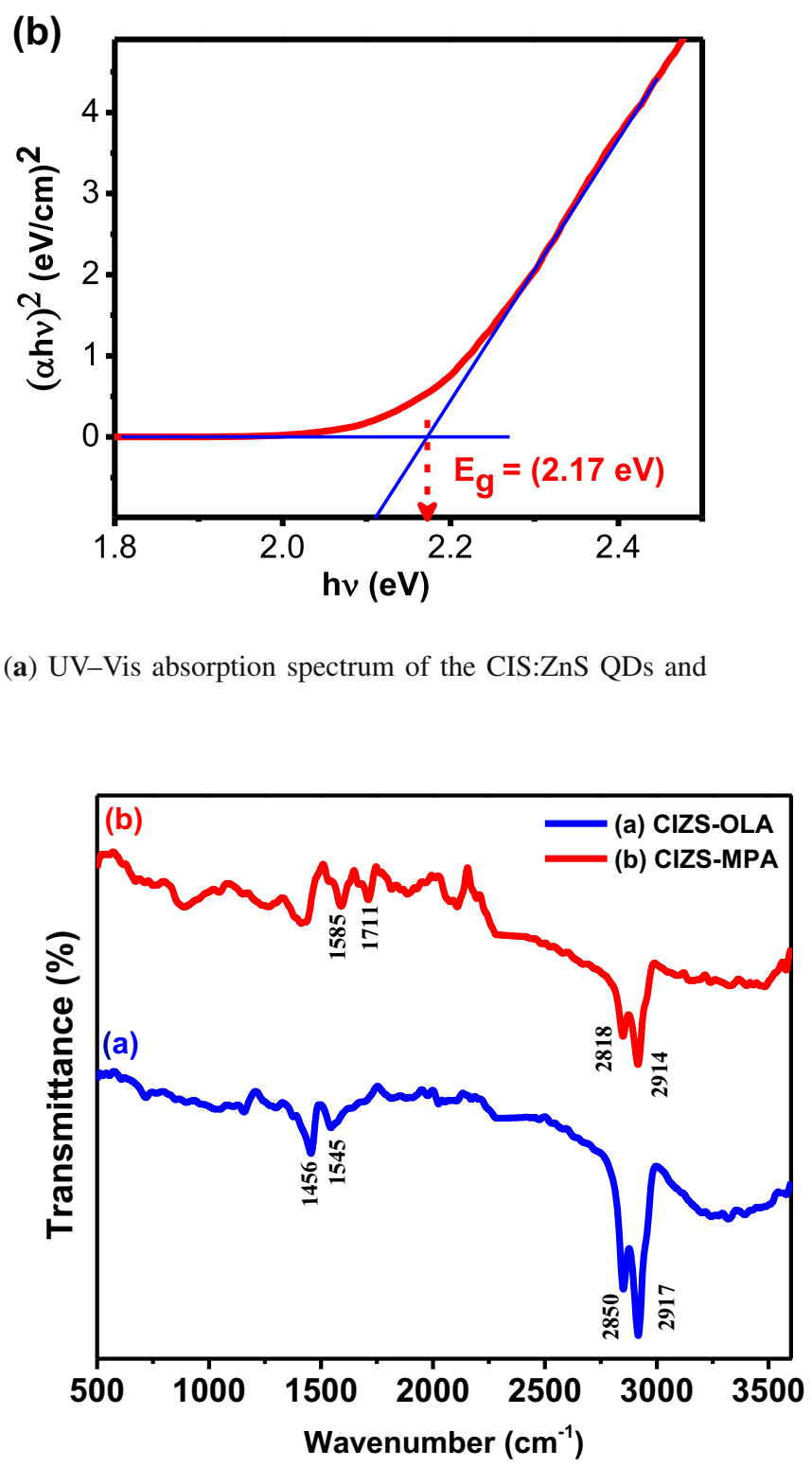

Figure 3. FTIR spectra of OLA- and MPA-capped CIS:ZnS QDs.

energy of the CIS:ZnS QDs. The optical band gap energy of the prepared colloidal QDs was estimated to be $\sim 2.17 \mathrm{eV}$ from Tauc's plot (figure 1b). The PL emission spectrum of the CIS:ZnS QDs (figure 2) shows high intense emission with the peak maximum nearly at $650 \mathrm{~nm}$. The band gap energy and the optical properties of the CIS:ZnS QDs promise their application in the next generation solar cell [2]. In order to confirm the ligand exchange process on the CIS:ZnS QDs, FTIR spectra of the native long chain ligand (OLA)-capped CIS:ZnS QDs and the ligand-exchanged (MPA capped) QDsensitized photoanodes were recorded.

The FTIR spectra of photoanodes with ligand-exchanged and the native ligand-capped QDs are shown in figure 3. The vibrational assignments of OLA from the passivating capping 


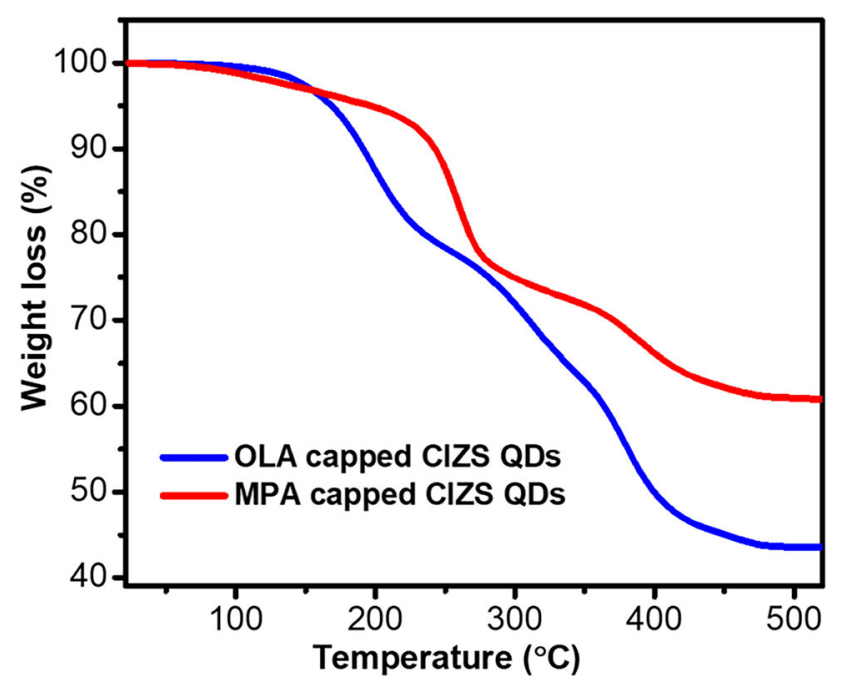

Figure 4. TGA of the CIS:ZnS QDs (OLA) and ligand-exchanged CIS:ZnS QDs (MPA).

layer over the CIS:ZnS QD-sensitized photoanodes (figure 3a) show prominent vibrational peaks at 1456 and $1545 \mathrm{~cm}^{-1}$, which are assigned to the bidentate $(-\mathrm{COO}-\mathrm{M})$ of the OLA capping ligand. The major intense peak was observed at 2850 and $2917 \mathrm{~cm}^{-1}$ which are assigned to the symmetric and asymmetric stretching vibrations of surface-capped OLA [15]. In the case of ligand-exchanged (MPA capped) QD photoanode (figure $3 \mathrm{~b}$ ), the prominent vibrational peaks were observed at 2818 and $2914 \mathrm{~cm}^{-1}$, which are from the stretching vibrational modes of aliphatic $(\mathrm{C}-\mathrm{H})$ groups present in the MPA $[16,17]$.

But, these vibrational modes are indistinguishable from the OLA bidentate peak. However, the presence of a less intense peak at $1711 \mathrm{~cm}^{-1}$ is from the asymmetric stretching of the (-COO-) vibrational mode which confirms the ligand exchange of MPA over the OLA-capped QDs. The low intense peak reveals that MPA was bound with the surface of CIS: ZnS QDs. Hence, the results suggest the partial ligand exchange of MPA over the OLA-capped QDs [18,19]. The solar cells were fabricated by sandwiching the CIS:ZnS QD-sensitized $\mathrm{TiO}_{2}$ photoanode and copper sulphide counter electrode. Two batches of QDSSCs labelled as QDSSCs with (MPA capped) and without ligand exchange (OLA capped) were fabricated to understand the influence of ligand exchange on the solar cell performance.

TGA has been carried out for the further confirmation of the ligand exchange process. TGA was performed for the OLAcapped and MPA-capped QDs under a nitrogen atmosphere from room temperature to $600^{\circ} \mathrm{C}$. The traces of TGA of the CIS:ZnS QDs before and after ligand exchange are shown in figure 4. The OLA-capped QDs show two-step mass reduction with the onsets around 180 and $320^{\circ} \mathrm{C}$ with the total weight loss of $56 \%$. The boiling point of $1-\mathrm{OCT}$ is $190^{\circ} \mathrm{C}$ and the boiling point of OLA is $360^{\circ} \mathrm{C}$. Hence, this weight loss around 180 and $360^{\circ} \mathrm{C}$ was attributed to the loss of residual organic solvents and ligands (1-OCT and OLA). The TGA curve of OLA-capped QDs shows major weight loss ( $\sim 50 \%$ from the original weight) around $280-400^{\circ} \mathrm{C}$, which reveals that the QDs are capped completely by OLA. On the other hand, the ligand-exchanged (MPA capped) CIS:ZnS QDs exhibit three-step mass reduction with the onsets of mass loss around 105,190 and $360^{\circ} \mathrm{C}$. An initial mass loss of about $8 \%$ in the region of $105^{\circ} \mathrm{C}$ was attributed to the MPA ligands (boiling point of MPA is $110^{\circ} \mathrm{C}$ ). The later mass reductions (at 190 and $360^{\circ} \mathrm{C}$ ) are due to the minimal residuals of 1OCT and OLA. Moreover, the total mass loss was found to be $39 \%$ only, which is almost half the amount of OLA-capped CIZS QDs. Hence, these results confirm the successful ligand exchange of long chain OLA to short chain MPA-capped CIS:ZnS QDs. And the results are in accordance with the FTIR analysis.
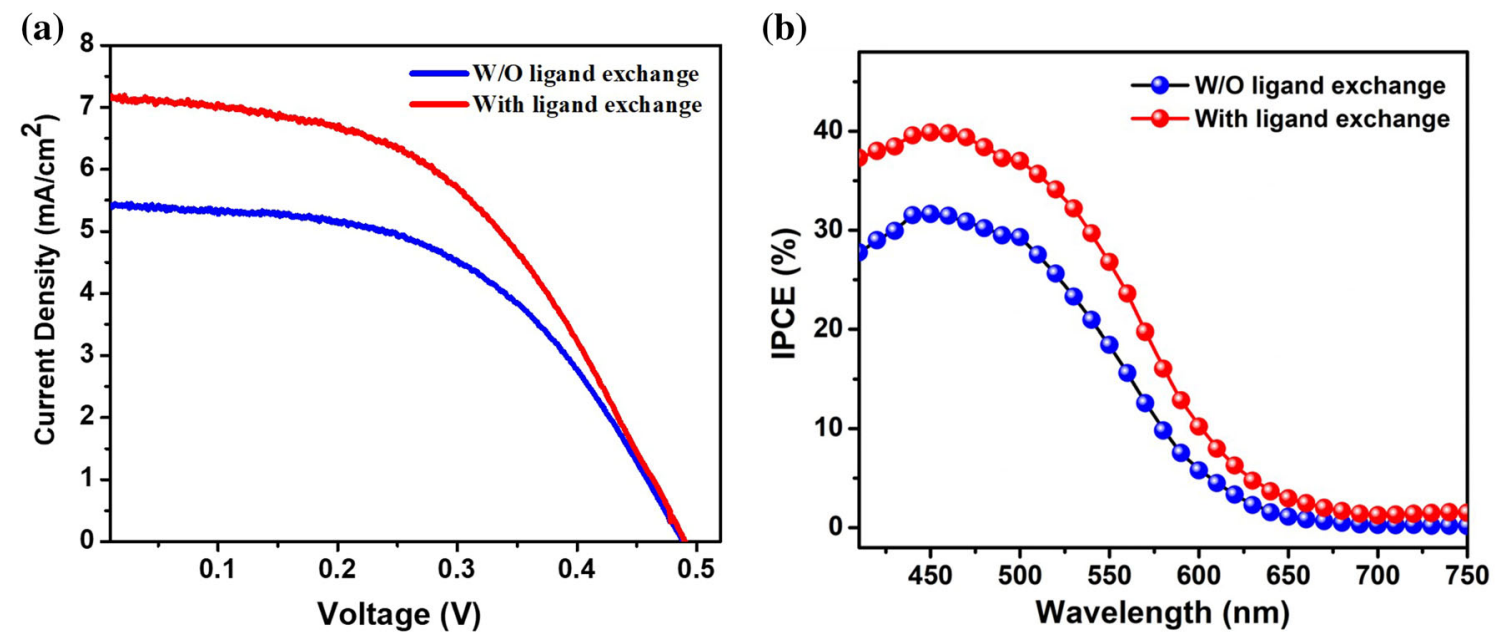

Figure 5. Photovoltaic characterization of QDSSCs. (a) $J-V$ characteristics of CIS:ZnS QDSSCs and (b) IPCE measurement of QD solar cells. 
Table 1. The photovoltaic properties of the solar cells.

\begin{tabular}{lccrr}
\hline Cell type & $V_{\mathrm{oc}}(\mathrm{V})$ & $J_{\mathrm{sc}}\left(\mathrm{mA} \mathrm{cm}^{-2}\right)$ & Fill factor & Efficiency $(\%)$ \\
\hline CIZS QDSSCs without ligand exchange & $0.492 \pm 0.002$ & $6.53 \pm 0.2$ & $0.46 \pm 0.03$ & $1.49 \pm 0.05$ \\
CIZS QDSSCs with ligand exchange & $0.494 \pm 0.007$ & $7.05 \pm 0.32$ & $0.49 \pm 0.02$ & $1.7 \pm 0.09$ \\
\hline
\end{tabular}

The photovoltaic performance of the fabricated QD solar cells was analysed by $J-V$ characterization and IPCE measurements (figure 5). The native ligand-capped (OLA) QD sensitized solar cell shows a power conversion efficiency of $\sim 1.5 \%\left(V_{\mathrm{oc}}=0.49 \mathrm{~V}, J_{\mathrm{sc}}=6.5 \mathrm{~mA} \mathrm{~cm}^{-2}\right.$ and Fill factor $=$ $0.46)$. The mean power conversion efficiency of the ligandexchanged (MPA capped) QD-sensitized solar cells was calculated as $\sim 1.7 \%$ from the $J-V$ characteristics, the photovoltaic parameters are evaluated as $V_{\mathrm{oc}}=0.492 \mathrm{~V}, J_{\mathrm{sc}}=$ $6.53 \mathrm{~mA} \mathrm{~cm}^{-2}$ and Fill factor $=0.46$. In both the QDSSCs, the open circuit voltage is almost the same; the improvement in the solar cell efficiency was only due to the enhanced short circuit current density $\left(J_{\mathrm{sc}}\right)$. The photovoltaic parameters are highlighted in table 1.

This implies the influence of ligand-exchanged QDs on the solar cell performance attributed by the increased charge carrier transport. The IPCE measurement (figure 5b) of the prepared solar cells supports the result of $J-V$ characterization. It was observed that ligand-exchanged QDSSCs show improved current conversion efficiency compared to the CIS:ZnS QDSSCs with native ligands. The results suggest that the enhancement in photovoltaic performance of CIS:ZnS QDSSCs is due to the improved charge carrier transportation and efficient light harvesting behaviour of the ligand-exchanged QDs.

\section{Conclusion}

The environmentally benign CIS:ZnS QDs were successfully synthesized by the solution-processed colloidal method using OLA as the capping ligand. The optical properties of the as-synthesized QDs were analysed through UV-Vis absorption and PL emission spectroscopy. The broad visible light absorption of the CIS:ZnS QDs was revealed from the absorption spectrum and the band gap energy was evaluated to be $\sim 2.17 \mathrm{eV}$. An inexpensive and heavy metal free CIS: $\mathrm{ZnS}$ QDs were employed as a less toxic photo-absorbing material in solar cells. The native long chain ligand (OLA) which stabilizes the QDs has been replaced with a small chain ligand (MPA) and their impact on the solar cell performances was analysed. The ligand exchange process was further confirmed through FTIR and TG analyses. The results reveal the partial ligand exchange of native long chain OLA-capped CIS:ZnS QDs with short chain MPA ligands. The photovoltaic characterization result implies that there is an enhancement in the solar cell performance of the ligand-exchanged
QD-sensitized solar cells. The photovoltaic parameters of the ligand-exchanged CIS:ZnS QD (MPA capped)-sensitized solar cells show a significant increase in the current density $\left(J_{\mathrm{sc}}\right)$ compared with OLA-capped CIS:ZnS QDSSCs. But there was no significant change in $V_{\mathrm{oc}}$ in both the cases. The long chain ligands capping over the QDs might have hindered the charge carrier transportation which causes a reduced photocurrent density than the ligand-exchanged QDSSCs. The IPCE measurements concurred with the results of solar cell efficiency that the IPCE values were increased considerably for the ligand-exchanged QDSSCs.

\section{Acknowledgements}

The authors sincerely thank DST (DST/TMC/SERI/FR/90), Govt. of India, for providing financial support. M Senthilkumar sincerely thank the Department of Science and Technology (DST), Govt. of India, for providing fellowship under (DST-INSPIRE) scheme and also thank Prof Prashant Kamat and Fulbright-Nehru fellowship for the short-term research at University of Notre Dame, USA.

\section{References}

[1] Han Z and Eisenberg R 2014 Acc. Chem. Res. 472537

[2] Chistyakov A A, Zvaigzne M A, Nikitenko V R, Tameev A R, Martynov I L and Prezhdo O V 2017 J. Phys. Chem. Lett. 8 4129

[3] Ma Q and Su X 2011 Analyst 1364883

[4] Choi M K, Yang J, Hyeon T and Kim D-H 2018 NPJ Flex. Electron. 210

[5] Yang D, Lu C, Yin H and Herman I P 2013 Nanoscale 57290

[6] Martynenko I V, Litvin A P, Purcell-Milton F, Baranov A V, Fedorov A V and Gun'ko Y K 2017 J. Mater. Chem. B 56701

[7] Zhang Y, Xiu W, Sun Y, Zhu D, Zhang Q, Yuwen L et al 2017 Nanoscale 915835

[8] Zhou Y, Zhao H, Ma D and Rosei F 2018 Chem. Soc. Rev. 47 5866

[9] Wang Y, Hu R, Lin G, Roy I and Yong K-T 2013 ACS Appl. Mater. Interfaces $\mathbf{5} 2786$

[10] Stroyuk O, Raevskaya A and Gaponik N 2018 Chem. Soc. Rev. 475354

[11] Zaiats G, Kinge S and Kamat P V 2016 J. Phys. Chem. C 120 10641

[12] Donegá Cd M 2011 Chem. Soc. Rev. 401512 
[13] Jara D H, Yoon S J, Stamplecoskie K G and Kamat P V 2014 Chem. Mater. 267221

[14] Carey G H, Abdelhady A L, Ning Z, Thon S M, Bakr O M and Sargent E H 2015 Chem. Rev. 11512732

[15] Bu W, Chen Z, Chen F and Shi J 2009 J. Phys. Chem. C 113 12176

[16] Vo N T, Ngo H D, Vu D L, Duong A P and Lam Q V 2016 J. Nanomater. 20161
[17] Maulu A, Rodríguez-Canto P J, Navarro-Arenas J, Abargues R, Sanchez-Royo J F, Garcia-Calzada R et al 2016 RSC Adv. 6 80201

[18] Jiménez-Hernández L, Estévez-Hernández O, HernándezSánchez M, Díaz J A, Farías-Sánchez M and Reguera E 2016 Colloids Surf., A 489351

[19] Wu Z, Li Y, Zhang L, Zhong Y, Xu H, Mao Z et al 2017 RSC Adv. 720147 